\title{
LATERAL SURFACE ELECTRIZATION OF Z-CUT LITHIUM NIOBATE DURING PYROELECTRIC EFFECT
}

\author{
A. N. Oleinik, ${ }^{1,2}$ P. V. Karataev, ${ }^{1}$ A. A. Klenin, ${ }^{2}$ \\ A. S. Kubankin, ${ }^{2,3}$ K. V. Fedorov, ${ }^{1,4}$ and A. V. Shchagin ${ }^{2,5}$
}

UDC 537.12, 537.13, 538.956

\begin{abstract}
The paper focuses on the surfaces electrization of Z-cut single-crystal lithium niobate during the pyroelectric effect. It is shown that the properties of the electric current passing through the surfaces of single-crystal lithium niobate depend on the ground circuit, the rate of temperature change, and orientation of polar axes. Experiments show that grounding of lithium niobate surfaces has an effect on the X-ray radiation during the pyroelectric effect.
\end{abstract}

Keywords: pyroelectric effect, lithium niobate, ferroelectric materials.

\section{INTRODUCTION}

Single-crystal lithium niobate $\left(\mathrm{LiNbO}_{3}\right.$ or $\left.\mathrm{LN}\right)$ is rather interesting to researchers due to its multiple physical effects (pyroelectric, electrooptic) that can occur in certain conditions [1]. Therefore, this material is extremely widely used, for example, in various electrooptical devices [2], infrared radiation measurements [3], as memory devices [4], in manipulating objects of about $1 \mu \mathrm{m}$ size [5], and others.

Additionally, LN single crystals can be used for generation and acceleration of electron and ion beams [6] and the X-ray generation $[7,8]$. These amazing opportunities arise from the pyroelectric effect in vacuum conditions, which is the ability of LN single crystals to generate an electric potential when they are heated or cooled. The generated electric charge is the source of the electric field in which electrons emit and accelerate due to ferroelectric electron emission and field-induced ionization. These electrons produce bremsstrahlung X-rays in the single crystal or other elements around (target, vacuum chamber walls).

However, studies of the role and influence of the lateral surfaces of the LN single crystal on the electric field generation during the pyroelectric effect, the electron emission and acceleration and the X-ray radiation, are still lacking. Geuther [9] and Nagaychenko [10] show that with increasing thickness of the single crystal (up to 10-15 mm) the generated electric potential increases. Hence, the increase in the size of the crystal surface has a positive effect on the above processes. In addition, Geuther [9] and Gillich [11] report about the current leakage through the LN surface at a temperature close to room, although the electrical conductance of lithium niobate is rather low in this temperature range $[1,12]$.

${ }^{1}$ John Adams Institute at Royal Holloway, University of London, Egham, UK, e-mail: Andrey.Oleinik.2017@live.rhul.ac.uk; Pavel.Karataev@rhul.ac.uk; Kirill.Fedorov.2016@live.rhul.ac.uk; ${ }^{2}$ National Research Belgorod State University, Belgorod, Russia, e-mail: starscream_46@inbox.ru; kubankin@bsu.edu.ru; ${ }^{3}$ Lebedev Physical Institute of the Russian Academy of Sciences, Moscow, Russia; ${ }^{4}$ National Research Tomsk Polytechnic University, Tomsk, Russia; ${ }^{5}$ Kharkov Institute of Physics and Technology, Kharkov, Ukraine, e-mail: shchagin@kipt.kharkov.ua. Translated from Izvestiya Vysshikh Uchebnykh Zavedenii, Fizika, No. 1, pp. 107-113, January, 2020. Original article submitted June 13, 2019. 


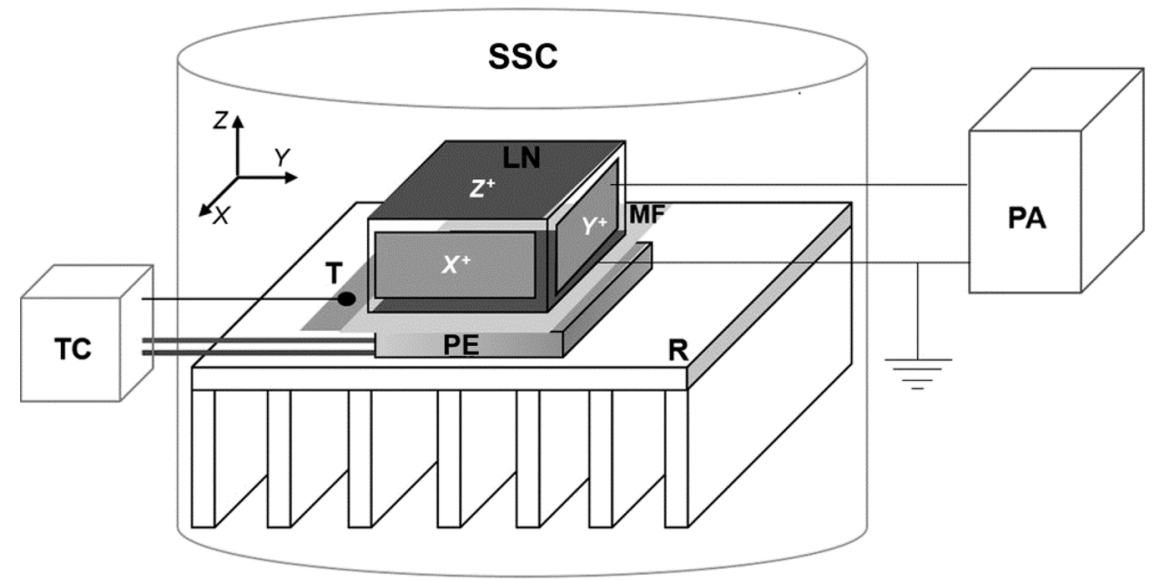

Fig. 1. Measuring circuit of short-circuit current between $Y^{+}$surface and $Z^{-}$surface grounded via the picoammeter. LN - lithium niobate, PE - Peltier element, T thermocouple, R - radiator, MF - Mylar foil, PA - 6485 Picoammeter, TC ATEC302 temperature controller, SSC - stainless-steel chamber.

However, to the authors' best knowledge no positive information is available in the literature about the charge on non-polar surfaces of the LN crystal observed during the pyroelectric effect, and the influence of the state of the LN surfaces on the X-ray radiation remains essentially unstudied. This work aims to contribute to closing this gap.

\section{ELECTRIC CURRENT MEASUREMENTS}

$Z$-cut crystals of lithium niobate in the form of $20 \times 20 \times 10 \mathrm{~mm}$ parallelepipeds were used for investigations. The crystals were grown in Tananaev Institute of Chemistry of Kola Research Centre of the Russian Academy of Sciences (Russia). The crystal specimens were cut such that the mechanical $X$ and piezoelectric $Y$ axes were normal to the crystal surfaces denoted as $X^{+}, X^{-}, Y^{+}, Y^{-}$. The latter formed the right-handed coordinate system with the polar surfaces $Z^{+}$and $Z^{-}$. It was believed that $X$-cut surfaces did not contribute to the charge generation, because any charge shift caused by an external force along this axis did not affect the other surface and caused neither pyroelectric nor piezoelectric effect, whereas an external force along $Y$-axis might cause piezoelectricity [13].

The electrostatic charge of the NL crystal surfaces was explored via the electric current measurements using a single-channel 6485 Picoammeter (Keithley Instruments, USA). The measuring circuit for the electric current is shown in Fig. 1. The aluminum foil $20 \mu \mathrm{m}$ thick was adhered to each crystal surface with electroconductive epoxy glue. In Fig. 1, one can see $X^{+}, Y^{+}, Z^{+}$surfaces with the aluminum foils which covered the entire area of each side, except for the outer contour $2 \mathrm{~mm}$ thick. The latter provided the current measurements only from the surface of interest and no charge effect from the adjacent surfaces. The temperature of the LN crystal was measured with a $\mathrm{K}$ type thermocouple mounted near one of the polar crystal surfaces. The crystal temperature was varied by a Peltier element supplied by an ATEC302 temperature controller. The measuring circuit included the LN single crystal, Peltier element, radiator, cooler, thermocouple, measuring foils on the crystal surfaces, and Mylar foil $5 \mu \mathrm{m}$ thick, which is insufficient for thermal insulation of the LN crystal from Peltier element. Mylar foil was used for electric insulation of the thermocouple from the picoammeter measurement line and placed in the stainless-steel chamber to eliminate external influences. The maximum value of the dark current in the measuring circuit was $5 \mathrm{pA}$.

Due to only one channel of the picoammeter, each crystal surface is measured separately, but almost at the same temperature conditions. Measurements of the electric current on the LN crystal surfaces are presented in the section below together with the measuring results obtained for different ground circuits and heating rates of $Z$-cut surfaces. 


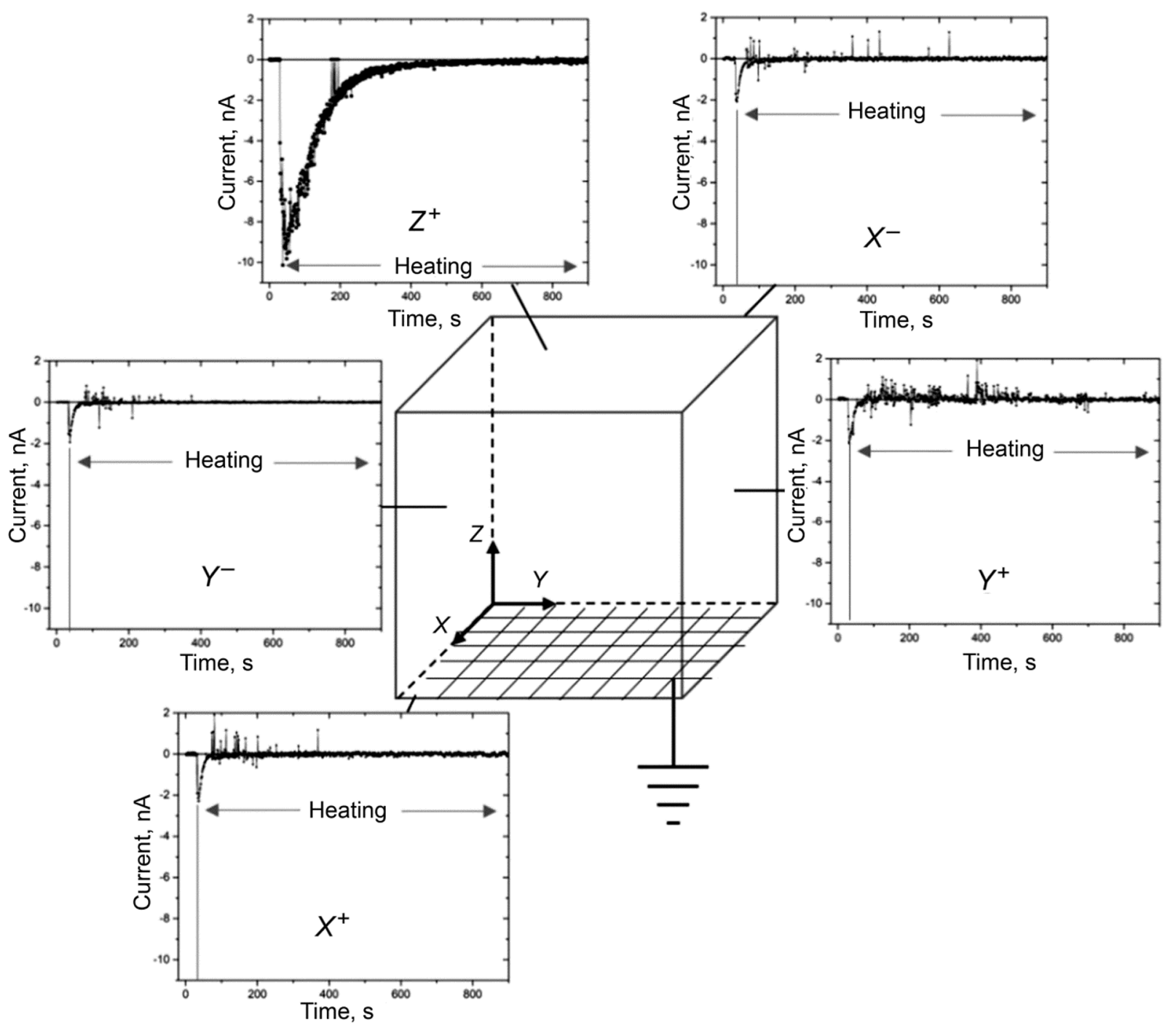

Fig. 2. Time dependences of short-circuit current between $Z^{-}$surface and the remaining five crystal surfaces in heating conditions.

\section{MEASURING RESULTS OF ELECTRIC CURRENT ON CRYSTAL SURFACES}

\section{First measurements}

First current measurements are performed in heating the $\mathrm{LN}$ crystal for $900 \mathrm{~s}$ at constant parameters of power supply of a group of Peltier elements. In each measurement, the crystal temperature changes from $(25 \pm 3)$ to $(55 \pm$ $3)^{\circ} \mathrm{C}$. Pyroelectric current is measured on the polar $Z^{+}$surface and then at each crystal surface individually. In all measurements, $Z^{-}$surface is grounded through the picoammeter, as shown in Fig. 1. The measurement results are presented in Fig. 2.

According to Fig. 2, in measuring the current flowing through the lateral surfaces $X^{+}, X^{-}, Y^{+}, Y^{-}$, a negativepolarity primary current pulse occurs, which is much shorter and weaker than the similar pulse of the pyroelectric current on the polar surface $Z^{+}$induced by the temperature change. After the drop of the primary current pulse, short 


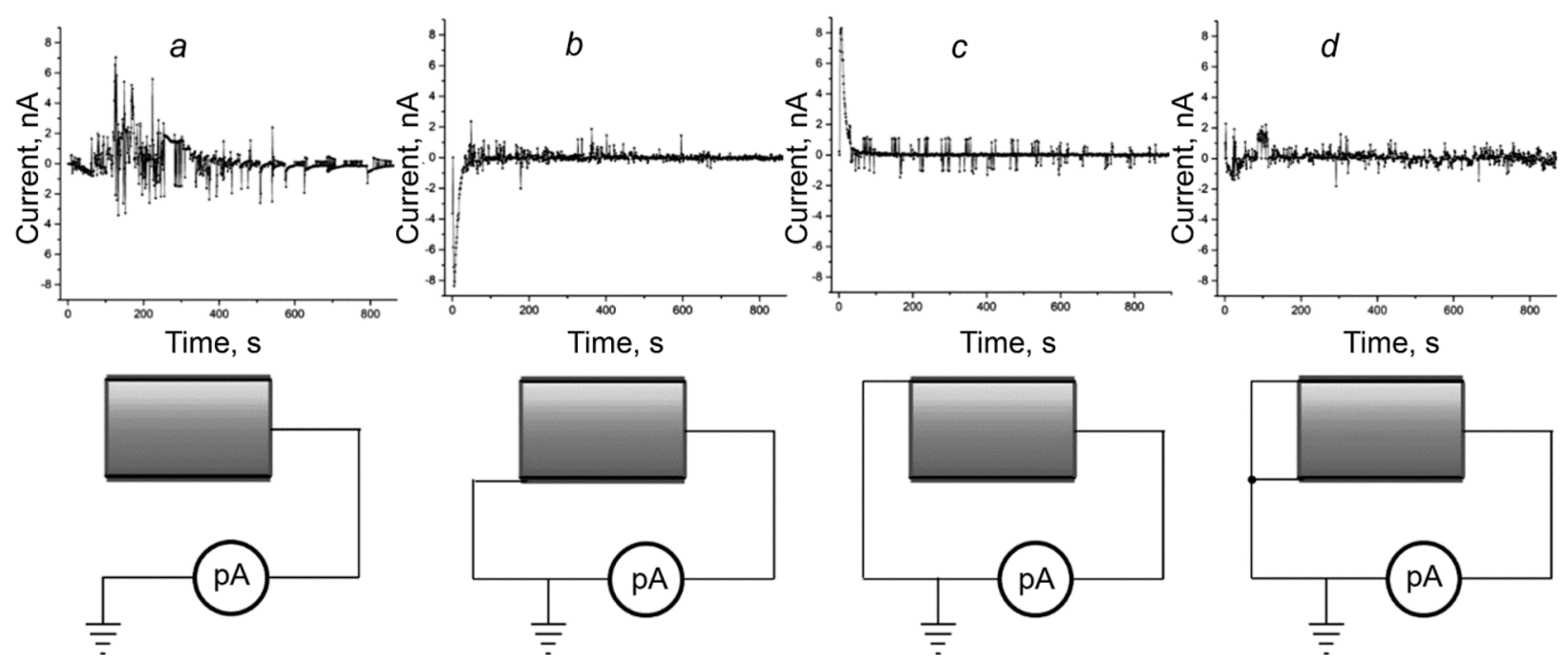

Fig. 3. Current curves obtained with different ground circuits for polar crystal surfaces: $a$ - no ground, $b$ - grounded lower surface $Z^{+}, c$ - grounded upper surface $Z^{+}, d$ - grounded both surfaces.

secondary current pulses (not over 3-5 s) periodically occur on the lateral surfaces. At the same time, the primary pulse of electric potential on the polar $Z^{+}$surface gradually decreases due to the change in the crystal temperature. The same polarity of the current recorded on all the surfaces at the beginning of heating, suggests that the current leaks out through the lateral surfaces from the polar to the grounded crystal surface. This leakage current weakens during 50$60 \mathrm{~s}$, when about $25-35 \%$ of the charge generated on the polar surface passes through the lateral surfaces. Subsequent secondary current pulses on the lateral surfaces are more unexpected, but repeated measurements and the measuring circuit testing confirm that this phenomenon is reproducible and not associated with electrostatic interference or instrument errors. It is also worth noting that on average, the secondary pulse intensity on positive $X$ - and $Y$-cut surfaces is stronger than on the negative, the current intensity on $Y$-cut surface being $25-30 \%$ higher than on the $X$-cut.

\section{Current measurements by different ground circuits}

The next stage includes current measurements via all possible ground circuits for Z-cut surfaces of the LN crystal using one and the same thermal influence in order to verify the assumption that the primary pulse is the leakage current. In Fig. 3, one can see the total current curves with the respective ground circuits obtained for all the lateral surfaces during the crystal heating. Moreover, the possible contribution of the current flowing through the gap between the crystal and the metal chamber is also considered. It is shown that the primary current pulse polarity changes with the change in the grounded surface, while the primary current pulse polarity on the lateral surfaces always coincides with that of the pyroelectric current on the ungrounded polar surface. In the case of no ground, intensive secondary current pulses of both polarities are observed. And in the case of grounding both polar surfaces, the intensity of secondary current pulses drastically lowers, and the primary current pulse does not exceed the value of the secondary.

These facts allow us to suppose that there are two current sources on the non-polar surfaces of lithium niobate, viz. the current leaking out from one polar surface to the other and short secondary current pulses of different polarity. In the first case, when one of the surfaces is grounded (as in the case of pyroelectric X-ray generators), this contribution predominates, and during the first $50-60 \mathrm{~s}$ of the thermal influence manifests itself as a continuous primary current pulse. In the second case, secondary current pulses tend to be more prominent after the reduction in the leakage current and continue throughout the thermal influence. The nature of the second current source remains as yet unclear. Since this phenomenon occurs on all non-polar crystal surfaces regardless of the crystal-axis orientation, and the fact that both 


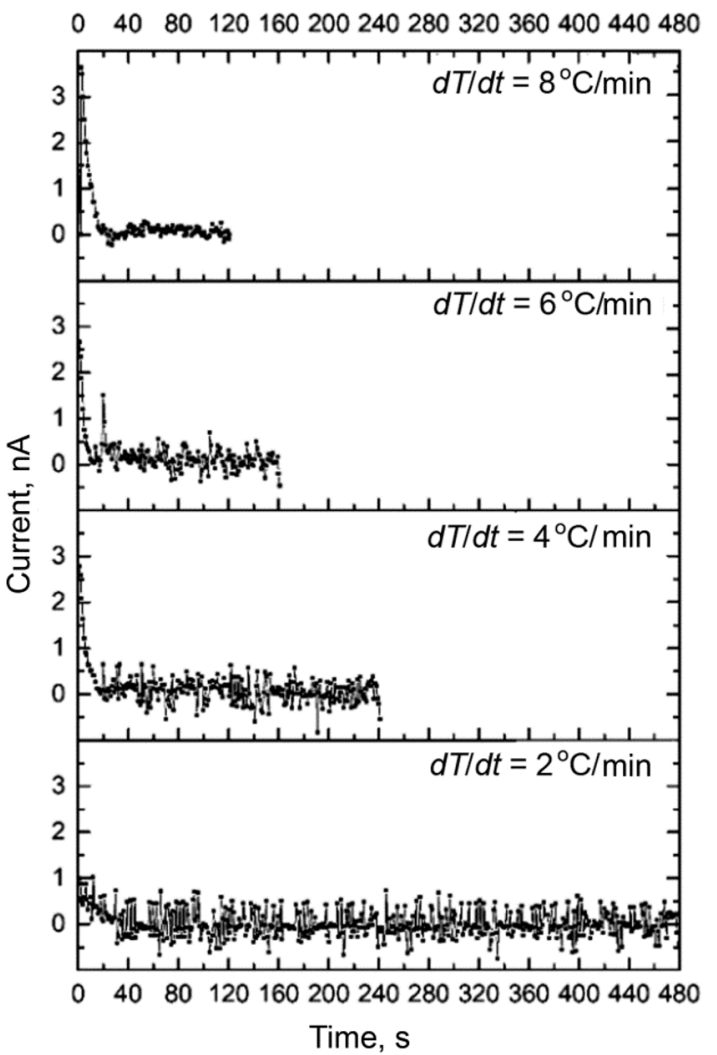

Fig. 4. Time dependences for the current between $X^{-}$surface and grounded $Z^{-}$surface obtained at different rates of temperature change.

current polarities manifest themselves equally, we can conclude that the observed current pulses are not connected with piezoelectricity and the temperature-induced changes in the crystal size.

\section{Current measurements on crystal surfaces at different rates of temperature change}

The electric current is measured on $Z^{+}$surface and all lateral surfaces (with the grounded $Z^{-}$surface) at different rates of the crystal temperature change to more carefully investigate the secondary current pulses of unknown origin. Measurements are made at 2, 4, 6 and 8 degrees Celsius per minute at a $15^{\circ} \mathrm{C}$ temperature change. Time dependences are shown in Fig. 4 for the current between $X^{-}$surface and grounded $Z^{-}$surface. The results for the other surfaces are identical. It is worth noting that the measurement time decreases with increasing rate of temperature change (because the given temperature difference is achieved faster); the sampling frequency is $1 \mathrm{~Hz}$ for all measurements.

At linear heating, the time dependence generally remains the same. First of all, we observe the primary current pulse of the polarity similar to that of the pyroelectric current on the ungrounded polar surface. Next, secondary pulses of different polarity occur. Of particular importance is the fact that the pulse intensity lowers as the rate of temperature change increases. When it grows from 2 to $8 \mathrm{deg} \mathrm{C}$ per min, the average current generated by secondary pulses decreases by 2 times.

This behavior shows that secondary current pulses relate to the thermal influence on the LN single crystal. They probably originate from piezoelectricity caused by thermally induced mechanical stresses in the crystal inhomogeneities. The latter occur at partial mechanical pressing of one of the crystal surfaces (here $Z$-cut surface) and are implicated in single responses directed opposite to the main orientation axis of the free crystal (thermal piezoelectricity) [14]. With increasing temperature change rate, mechanical stresses probably have no time to occur in 

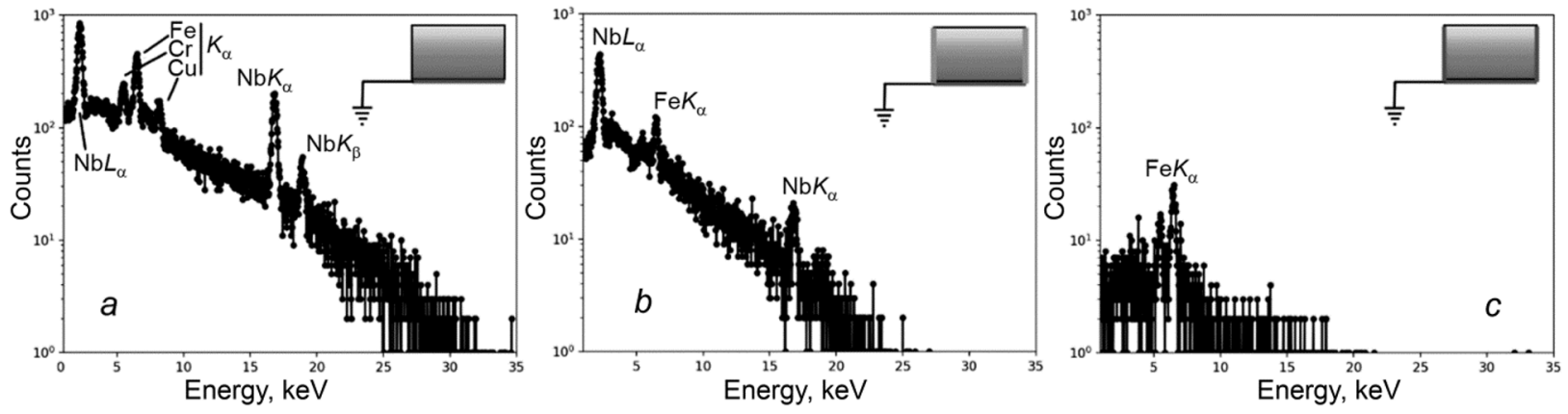

Fig. 5. X-ray spectra measured at different states of the LN surface: $a$ - uncoated, $b$ - coated by dielectric adhesive, $c$ - insulated with grounded aluminum foil.

sufficient amount during the current observations. More research into the current generation from the crystal surfaces is still necessary for the full identification of the described phenomenon, in particular, during the combined mechanical and thermal influence on the LN crystal.

\section{LATERAL SURFACE EFFECT ON X-RAY GENERATION}

Earlier the common belief was that only the polar surface of pyroelectric crystals charged, and during their temperature change in vacuum, the electron emission and acceleration occurred owing to it only. The obtained results showed that $Z$-oriented surfaces of lithium niobate also possessed electrostatic charge that needed to be considered. It was logical that leakage currents caused the charge losses from the crystal polar surface and, consequently, lowered the efficiency of pyroelectric X-ray generators. At the temperature change rate of about $8 \mathrm{deg} \mathrm{C}$ per min, the X-ray emission was maximum for a cylindrical sample of lithium tantalum [15]. As was shown above, at that rate of the temperature change and in conditions close to the experimental [15] (but for another sample shape), the surface current pulses were the lowest. This fact inspired us to explore how the electrostatic charge on the crystal surfaces affected the $\mathrm{X}$-ray generation during the pyroelectric effect.

The X-ray spectra in Fig. 5 are measured for different states of the lateral surfaces subjected to the temperature change in vacuum conditions, namely uncoated surface, surface coated by dielectric adhesive and surface insulated with grounded aluminum foil. The upper part of the lateral surface $2 \mathrm{~mm}$ thick adjacent to the ungrounded $Z$-cut surface, is not covered at all to avoid the increase in the leakage current. The dielectric adhesive coating provides the pulsed current screening, such that the lateral surfaces do not contribute to the electric field generation. Grounding of the lateral surfaces with the aluminum foil completely eliminates their electrization.

The X-ray emission was measured by an X-ray detector using a cadmium telluride $(\mathrm{CdTe})$ semiconductor (Amptek Inc., USA). The detector was mounted to the upper flange of the vacuum chamber, in front of the polar crystal surface. When measuring the X-ray spectra, the polar surface near the detector was free of foil or glue. All measurements were performed during a heating and cooling cycle of the LN crystal in the temperature range of 25 $45^{\circ} \mathrm{C}$ and $\sim 1$ mTorr residual gas pressure.

Any attempts to modify the crystal surface lead to a drastic decrease in the X-ray generation during the pyroelectric effect. And in case of the grounded surface, the X-ray generation completely disappears at a positive charge on the polar surface. This indicates that the electrostatic charge on the crystal surfaces contributes much to the electric field generation during the pyroelectric effect in vacuum conditions. 


\section{CONCLUSIONS}

The experimental measurements clearly indicated to the presence of an electric charge on the lateral surfaces of $Z$-cut lithium niobate at its temperature changes. Two different charge sources were distinguished. First was the current leaked out from the ungrounded polar surface, which was the most noticeable in the first several tens of seconds of the thermal influence. Afterwards, the contribution of the leakage current was very weak. This behavior of the leakage current requires further investigation. Second were short current pulses of different polarity (usually not exceeding $1 \mathrm{nA}$ at each surface), which replaced the leakage current. Those pulses weakened with the increased temperature change rate. Their origin was not completely clear. Probably, that was manifestation of thermal piezoelectricity. The behavior of secondary pulses depending on the orientation of polar $X$ and $Y$ axes requires additional research.

It was shown that the charged lateral surfaces had a significant effect on the X-ray generation during the pyroelectric effect, that was not considered before and must be considered in developing pyroelectric X-ray generators. An attempt to shield or eliminate the lateral surface electrization resulted in a drastic decrease in the X-ray radiation. Electrization of the LN lateral surfaces during the pyroelectric effect must be investigated more closely in order to determine the nature of currents, explain the dynamics of the leakage current from the polar surfaces, and estimate this effect in various applications of single-crystal lithium niobate.

This work was supported by the government contract N 3.1631.2017/PCh from the Ministry of Higher Education and Science of the Russian Federation.

\section{REFERENCES}

1. K.K Wong, Properties of Lithium Niobate, UK, INSPEC, London (2002).

2. J. E. Toney, Lithium Niobate Photonics, UK, Artech House, London (2015).

3. J. G. Webster and H. Eren, Measurement, Instrumentation, and Sensors Handbook: Electromagnetic, Optical, Radiation, Chemical, and Biomedical Measurement, USA, CRC Press, New York (2014).

4. M. Sumets, Lithium Niobate-Based Heterostructures: Synthesis, Properties and Electron Phenomena, UK, IOP Publishing, London (2018).

5. M. Carrascosa, A. García-Cabañes, M., Jubera et al., Appl. Phys. Rev., 2, 040605 (2015).

6. J. A. Geuther and Y. Danon, J. Appl. Phys., 97, 074109 (2005).

7. J. D. Brownridge and S. M. Shafroth, http://arxiv.org/ftp/physics/papers/0311/0311090.pdf.

8. J. A. Geuther and Y. Danon, J. Appl. Phys., 97, 104916 (2005).

9. J. A. Geuther, PhD Thesis, Troy; Rensselaer Polytechnic Institute, New York (2007).

10. V. I. Nagaychenko, Poverkhnost'. Rentgenovskie sinkhrotronnye i neitronnye issledovaniya, 3, 81-89 (2007).

11. D. Gillich, PhD Thesis, Troy; Rensselaer Polytechnic Institute, New York (2009).

12. A. A. Esin, A. R. Akhmatkhanov, and V. Y. Shur, Ferroelectrics, 496, 102 (2016).

13. S. Sanna and W. G. Schmidt, J. Phys.: Condens. Matter, 29, 413001 (2017).

14. Y. M. Poplavko, A. I. Otkox, G. G. Krainyk, et al., in: Proc. 8th Int. Symp. on Electrets (ISE 8), Paris (1994), pp. 731-735.

15. A. S. Kubankin, A. S. Chepurnov, O. O. Ivashchuk, et al., AIP Adv., 8, 035207 (2018). 